\title{
UNA REFLEXIÓN ACERCA DE LA PERTINENCIA Y LA RELEVANCIA DE LA INVESTIGACIÓN
}

\author{
A REFLECTION ON THE RELEVANCE AND \\ PERTINENCE IN UNIVERSITY RESEARCH
}

Héctor M. Morán Seminario

\section{Resumen}

Este trabajo es un aporte a la discusión del concepto de pertinencia-relevancia de la investigación universitaria, mostrando sus diversas acepciones por la falta de consenso en su definición, lo que lleva a que en esta polisemia caracterizan concepciones de universidad y también de desarrollo que desembocan en que según la perspectiva que se dé al sentido de pertinencia genera cuestiones epistemológicas e ideológicas relacionadas con el para qué y para quién se hace y sirve la investigación científica que se hace en la universidad y como ayudarán a la solución de los problemas de la sociedad concreta. El grado de pertinencia depende por una parte de la importancia que el asunto tenga en la sociedad concreta, en su sobrevivencia, en su medioambiente, su cultura, su economía; se resalta que el nivel de pertinencia de la investigación depende del acumulado de conocimiento que tenga la Universidad para atacar el asunto.

Palabras clave: Pertinencia; Investigación científica; Universidad; Sociedad; Posgrado.

\section{Abstract}

This work is a contribution to the discussion of the pertinence concept of university research, showing its various meanings for the lack of consensus in its definition, which leads to that in this polysemy characterizes conceptions of 
university and also development that result, according to the perspective given to the sense of pertinence, generates epistemological and ideological issues related to for what and for whom university scientific research is made and is useful and how they will help to solve the problems of the concrete society. The degree of relevance depends in part on the importance of the issue in the concrete society, its survival, its environment, its culture, its economy; it is emphasized that the level of relevance of the research depends on the accrued knowledge that the University has to tackle the matter.

Key words: Relevance; Scientific research; University; Society; Postgraduate.

\section{INTRODUCCIÓN}

La esencia de la universidad radica en la producción y transferencia de conocimiento. Conocimiento que exige en todo momento ser revisado y debatido entre pares académicos. Un esfuerzo tanto individual como colectivo encaminado a la búsqueda inexhausta de lo verdadero y una forma de construir la conciencia histórica desde el ejercicio libre de la razón (Derrida, 2002).

Es conocida por todos la importancia que tiene fomentar la investigación científica. Pues una de las funciones fundamentales de la universidad es propiciar la creación de nuevos conocimientos a través de la investigación científica, tecnológica, humanística y social. Esta función se ha convertido en el centro de la evaluación de las universidades en muchos países del mundo, incluyendo el nuestro.

Sobre este asunto Acuña y Valenzuela (2016) nos dicen, que las universidades han pasado de ser depositarías del conocimiento y la cultura, a ser formadoras de cuadros profesionales, conscientes de su entorno y de lo que son capaces de hacer por él, para lo que requieren tener gran sensibilidad para orientar los acontecimientos que guiarán el rumbo de la sociedad, adelantarse a su tiempo y tener la capacidad de gestión que permita construir una sociedad con una mayor calidad de vida (http://148.231.9.38/JG/foro/ pdf/25.pdf).

Es por ello que para muchos estudiosos de la educación superior, la verdadera universidad debe estar inmersa en la actualización y generación de conocimiento, del cual, desde un enfoque integral e interdisciplinario, permita analizar y resolver los problemas que actualmente están presentes en la sociedad, así como proveer estrategias para la construcción de una sociedad más justa, responsable y solidaria, que sea capaz de erradicar la pobreza, las enfermedades, la violencia $\mathrm{y}$ dinamizar los avances tecnológicos (Acuña y Valenzuela: 2016, p.1).

Entrando al ámbito de la investigación en la universidad, continúan Acuña y Valenzuela (2016), la investi- 
gación, junto con la docencia y la extensión, es una de las funciones esenciales de la Educación Superior. Nosotros también creemos que lo sostenido por nuestros autores se corrobora en casi todas las universidades del mundo, basta revisar las correspondientes reglamentaciones universitarias. Y esto es así porque esta función responde al compromiso que tiene la universidad de ser un centro productor de conocimiento que sirve para dar respuesta a las múltiples situacionesproblema que se viven en cada país y para la creación, aplicación y transmisión de nuevo conocimiento.

Resaltando la importancia de la investigación, los autores establecen dos ámbitos en que su impacto es muy notorio, ya que la consideran como actividad fundamental generadora de conocimiento que se le puede considerar como el motor que articula tanto la formación y la docencia así como la extensión y la vinculación. Esto significa, que por una parte produce conocimientos que permiten actualizar y mejorar los planes de estudio, $y$, por otra, ofrece valiosos instrumentos para lograr un vínculo efectivo con la sociedad (p: 1). Asimismo, para los autores, la investigación debe abordar temas de conocimientos relevantes e importantes cuyos resultados podrán contribuir directamente con la prosperidad del conocimiento individual y colectivo. De lo que se desprende, "que la pertinencia de una investigación está relacionada con el grado de mejora del espacio social donde se integrarán los conocimientos adquiridos o los resultados de la misma” (Acuña y Valenzuela (2016, p: 2)

En este panorama contextual, la conciencia sobre la urgente necesidad de una educación superior con mayores niveles de pertinencia, es uno de los grandes aportes que hizo la Conferencia Mundial sobre Educación Superior convocada por la UNESCO y desarrollada en París del 5 al 8 de octubre en 1998.

En sentido amplio, la pertinencia, este concepto que es eje del presente artículo, depende de cómo se interviene en la situación, en nuestro caso, la investigación y la universidad. Cada entorno es punto de partida de lo pertinente, pero la consciencia es quien distingue y concede la cualidad de pertinencia a las entidades. Los recursos son pertinentes en la medida que son acordes con una voluntad intencionada.

Pero pertinencia e impertinencia son atributos de las acciones humanas y de sus instituciones. Si las actividades llevadas a cabo por las instituciones educativas son impertinentes, no contribuyen a preservar y enriquecer la cultura. Siendo impertinentes, las universidades no producen las capacidades cognitivas y laborales requeridas por la sociedad para su desarrollo. La investigación científica, de ser actividad impertinente, no genera los conocimientos valiosos y suficientes que mejoren las condiciones de vida, la conciencia colectiva y el sentido humano de las comunidades. Una universidad impertinente condena a 
su sociedad al extravio y contradice su misión fundamental. El gasto social aplicado a una universidad que incurre en impertinencias resulta infructuoso. La investigación científica al ser impertinente profundiza las condiciones de dependencia intelectual y tecnológica y desaprovecha las capacidades locales y regionales (Garrocho y Segura, 2012. On line).

Precisamente la relevancia/pertinencia de la investigación científica en la universidad es el núcleo del presente trabajo. Pretendemos echar luces y dar más claridad a esta categoría en el nuevo léxico de la educación superior. Para este propósito hemos realizado una exploración en internet de la literatura existente producida por académicos que han hecho de la pertinencia tema de sus reflexiones, crítica y esclarecimiento desde sus correspondientes posiciones epistemológicas, politicas, sociales y académicas, pero, sin que hasta la fecha se haya logrado un consenso sobre lo que es la relevancia/pertinencia, por ser un término polisémico. Nuestra contribución no reclama originalidad ni paternidad en la discusión, pues mi aporte se apoya en los autores que aparecen en el documento, si en algo se ha contribuido es a darle coherencia y sentido de precisión al discurso sobre este tópico y ponerlo en la agenda de la academia nacional.

El presente ensayo está dividido en dos partes: la primera aborda el problema de la pertinencia/relevancia en los aspectos teórico-conceptuales de la investigación científica en la universidad; la segunda, focaliza en la pertinencia/relevancia en la investigación realizada a nivel de tesis universitarias y describe la situación del posgrado.

\section{PARTE I}

¿De qué hablamos cuando hablamos de la pertinencia de las investigaciones?

\section{Los sentidos de la pertinencia/ relevancia}

Gonzales (2001), sobre el particular, nos dice que cada vez con más frecuencia, a quienes les corresponde la responsabilidad de la gestión de la investigación en las organizaciones de educación superior universitarias, se les exige que los trabajos de investigación que se auspicien, promueven, patrocinen y, principalmente, financien, sean pertinentes. Para Gonzáles esto significa que la pertinencia es uno de los criterios que han de considerarse cuando se evalúan las solicitudes de financiamiento para ejecutar proyectos de investigación; pero, al parecer, aún no existe claridad suficiente acerca del significado de este vocablo y sigue aún sin ser respondida la interrogante ¿qué es pertinencia? Estando también interesado en echar luces sobre esta categoría nos ofrece alcanzarnos algunas ideas esclarecedoras como una contribución a la búsqueda de una respuesta. En lo que sigue seguiremos a Gonzales, glosando y parafraseando lo más relevantes de su aporte.

En primera instancia, Gonzales hace una precisión muy importante: 
señala que la investigación es un quehacer que aun cuando sea llevado a cabo individualmente está contextualizada socialmente. Y que, en el marco de este contexto social situacional, podría ubicarse el esfuerzo por definir la pertinencia de la investigación. De donde se sigue que una investigación es pertinente en la medida que responde a las necesidades que se generan en el marco social que le sirve de contexto. A continuación del concepto elaborado, reconoce tres componentes: el autor (actor), la respuesta y la necesidad.

El primero hace referencia a quien lleva a cabo la investigación; el segundo se vincula con el aporte que se genera como consecuencia de la acción investigativa, y el tercero alude a los requerimientos de la sociedad como marco regulador donde se sitúa el accionar de los investigadores; es este último el elemento referencial crucial para definir la pertinencia. En este sentido se conciben como necesidades sociales las siguientes: (a) Necesidad de CREAR; (b) Necesidad de SABER; (c) Necesidad de HACER;

(d) Necesidad de RESOLVER (Gonzales, 2001, p.1).

Por tanto, para nuestro autor se considerará pertinente la investigación que en sus procesos y/o productos, en alguna medida, satisfaga alguna o todas las necesidades mencionadas. Ahora bien, como se dijo antes, la actividad investigativa es llevada a cabo por personas $\mathrm{y}$, éstas, pueden actuar individual o colectivamente. La instancia colectiva puede ser asumida institucional o socialmente; individualmente, cuando se actúa en el marco de alguna organización formalmente instituida; colectivamente, cuando se actúa simplemente desde una perspectiva de ciudadanía; resaltando que las necesidades sociales son tales en la medida en que dichos requerimientos impacten a los individuos, a las instituciones o a la colectividad social como un todo. Con esta argumentación inicial, Gonzales plantea tres tipos de pertinencia: Individual, Institucional y Social. Veamos cada una resumidamente:

La pertinencia individual hace referencia a aquellas investigaciones cuyos procesos o productos responden a la necesidad propia del investigador para alcanzar su autorrealización como individuo, a través de sus contribuciones teórico o prácticas que produzcan conocimiento nuevo (Necesidad de crear), incrementando su propio repertorio cognitivo (Necesidad de saber), generando modos de transformar en acciones los saberes (Necesidad de hacer) y ofreciendo soluciones a problemas (Necesidad de resolver). Por su lado la Pertinencia Institucional se patentiza cuando la creación, generación de saber, transferencia o resolución, es asumida corporativamente por alguna organización investigativa institucional, como universidad, Instituto, Centro, etc. Por último, la Pertinencia Social es aquella que se genera de lineamientos planteados por 
instancias nacionales, regionales o locales, como, por ejemplo, la Agendas en diferentes áreas planificadas por el Consejo Nacional de Ciencia y Tecnología (CONCYTEC) en el caso peruano, o la política de investigación generada por el Vicerrectorado de investigación de las Universidades.

\section{Relevancia y calidad de la investiga- ción universitaria}

Al concepto de pertinencia ahora adicionaremos el concepto de "calidad" en la investigación científica en la universidad, siguiendo los planteamientos de Judith Sutz (2014), quien en un enjundioso artículo "Calidad y relevancia en la investigación universitaria: apuntes para avanzar hacia su convergencia", considera su definición dificultosa, porque se trata de conceptos "evidentes por sî mismos", y, que al decir de Weber, raramente son pensados con claridad, por la misma razón de que aparecen como obvios (p. 1). La autora sostiene que, en el fondo, no son evidentes ni obvios como se cree, sino que "Un conjunto de apreciaciones potencialmente divergentes sobre dichos conceptos deriva de las respuestas a preguntas como calidad y relevancia para qué y para quién; otro conjunto tiene que ver con quién contesta legitimamente las preguntas anteriores" (p. 2), lo cual las hace aparecer como evidentes y obvios por sí mismos.

La investigadora nos señala con mucha precisión que el problema de la obviedad y lo evidente radica en que en el mundo académico no se hace una diferenciación explícita en la descripción de ambos términos. Es decir, que esta falta de precisión permite identificar una universidad y cualquier centro de investigación en lo referente a la calidad y la pertinencia en cuanto a la investigación. Pero, intuitivamente, esto no parece ser totalmente correcto. Porque si bien la calidad se puede valorar igualmente en todos los casos, su identidad con la pertinencia solo parecería ocurrir cuando se trata de instituciones académicas cuya función principal o única es hacer avanzar el conocimiento en general. En las instituciones donde se realiza investigación junto a otras funciones o con otros objetivos relacionados al avance del conocimiento, como es el caso de la universidad, la identidad entre calidad y relevancia "se desdibuja".

Sutz quiere decirnos que el "desdibujamiento" de la identidad mencionada significa la autonomización relativa de calidad y relevancia; hecho que se patentiza con especial claridad cuando la investigación es mirada con una perspectiva "no exclusivamente académica”, es decir, cuando además de pares, los que miran son "impares" (Citando a Avalos, 1997). Intentemos explicar esta mirada de los "impares". Nos dice Sutz, que concretamente en el caso de la investigación universitaria, "los impares son, en primera instancia, los que en diversas esferas -politica, productiva, social, culturalbuscan incorporar los resultados obtenidos a su reflexión y a su accionar." (P. 6). Es decir, que estos resultados 
de la investigación serán apreciados como relevantes por los diversos actores "impares", de acuerdo a variadas circunstancias, que incluyen la capacidad que tengan para utilizarlos, la utilidad más o menos directa que perciban que tienen para responder a sus inquietudes y problemas, el grado de participación que hayan tenido en la definición de los problemas a investigar y su involucramiento durante el proceso mismo de investigación (p. 7).

Por otro lado, Sutz señala que la legitimidad de la apreciación de relevancia de la investigación universitaria, en particular en las universidades públicas, por parte de los impares, tiene dos fuentes. Una es de carácter político, pues los poderes públicos financian la mayor parte de la investigación universitaria (que en otras realidades, que no es la nuestra, lo hacen en el marco de una politica general de desarrollo nacional), razón por la cual es válido que tengan expectativas de esta inversión, es decir, entre otras cosas, que redunde en respuestas adecuadas a los problemas de múltiples actores, incluidos los de los propios poderes públicos. De aquí se sigue que sea natural que se formen una opinión sobre su pertinencia/relevancia. Además, en la medida en que la financiación estatal de la investigación en las universidades públicas no define qué es lo que se investiga, la apreciación de relevancia por parte de los poderes públicos no es auto-referenciada (p. 3).

La segunda fuente de legitimidad de la mirada externa sobre la calidad y la relevancia de la investigación uni- versitaria, continua afirmando Sutz, deriva de razones propiamente académicas, pues las preguntas, criticas y preocupaciones del mundo extraacadémico constituyen, hoy como ayer, una de las orientaciones más fértiles para una investigación relevante y de calidad en la universidad (p. 3).

A manera de sintesis introductoria de su reflexión, la autora afirma que al poner la investigación universitaria en un marco social amplio, su calidad y su relevancia/pertinencia dejan de ser conceptos intrinsecamente vinculados y pasan a estar analíticamente diferenciados; hecho que abre la posibilidad de divergencias: investigación de calidad pero de relevancia discutible; investigación sobre temáticas relevantes pero de calidad discutible. Esto sirve para que la autora proponga que se deban referir los conceptos de manera más directa a objetivos de la investigación universitaria -no puede apreciarse ni calidad ni, sobre todo, relevancia, si no es en relación a objetivos- y también a la vinculación de ésta con diferentes actores que tienen razones para expresar expectativas en torno a ella. Pues ambos, calidad y relevancia, son conceptos asociados a construcciones sociales por la sencilla razón de que no hay calidad ni tampoco relevancia reveladas.

Ahora, retomando la afirmación hecha al inicio por la autora acerca de que los conceptos de calidad y pertinencia/relevancia no son evidentes ni obvios, sino que más son producto de puntos de vista ante las preguntas sobre para qué y para 
quiénes es pertinente la investigación. Al respecto, revisemos estas cuestiones siguiendo la argumentación de nuestra invitada:

\section{La relevancia de la investigación académica asociada al "para qué hacer investigación".}

Las respuestas a la pregunta "investigación para qué" fuera del ámbito académico, han tendido a concentrarse en dos aspectos centrales: crecimiento económico por una parte y toma de decisiones por otra, afirma Sutz, lo que en lo básico significa:

Que la investigación científica está en la base de los grandes cambios tecnológicos que desde mediados del siglo XIX han asegurado un crecimiento económico sostenido, al menos en los países altamente industrializados, es algo indiscutible. Esto significa que la expectativa de crecimiento económico es la que genera una respuesta válida al para qué dedicar recursos, en particular, recursos públicos, que permitan realizar investigación en las universidades públicas, como es el caso en nuestro medio.

Por otro lado, sigue refiriendo la autora que hay algunas áreas de actividad para las cuales la investigación científica resulta clave como apoyo a la toma de decisiones, aunque no siempre se tomen como receta infalible, porque también nunca faltan barreras que se levantan entre la investigación y la definición de políticas, así como los posibles peligros y desviaciones asociados a la influencia del conoci- miento experto (Nutley et al, 2007; Jasanoff, 1990; citados por Sutz, 2014, p. 5).

Con todo esto, considera que los resultados de la investigación constituyen sin duda un insumo importante $\mathrm{y}$, sobre todo, considerado imprescindible, al menos a nivel de discurso, para fundamentar porqué se proponen ciertos caminos y por qué otros son rechazados $(2014$, p. 5).

\section{"¿Para qué hacer investigación?": La demanda de nuevo conocimiento.}

En otro ángulo, la autora discurre sobre la relevancia respecto a la asociación entre relevancia de la investigación académica y la pregunta “¿para qué hacer investigación?” Su propuesta, es: por la demanda de nuevo conocimiento. Esto quiere decir, que la pertinencia/relevancia se materializa en la medida en que hay demanda. Dicho de otro modo: es razonable asumir que si existe una respuesta (positiva) a esa pregunta es porque algún actor concreto está demandando conocimiento; debiendo precisarse que esa demanda puede ser explícita y formulada ex ante, como cuando se contrata una investigación para resolver determinado problema o para responder a cierto interrogante. Puede también ser difusa y ex post, como ocurre cuando se reconoce la utilidad de un resultado de investigación existente para abordar cierto problema. Ambos tipos de demanda tienen como punto de partida la hipótesis de que la investigación puede contribuir a resolver problemas 
y a contestar preguntas; es decir, que es relevante (Sutz, 2014, p. 6).

Ahora, naturalmente que estamos pensando en una demanda solvente, pues será ésta la que tiene el poder de otorgar pertinencia/relevancia.

Veamos la otra cara de la moneda. Aceptando que Sutz está en lo correcto en la forma de presentar la cuestión y que tiene razones a favor, es válido preguntarse ¿qué ocurre con la relevancia de la investigación cuando la demanda de conocimiento, en particular la solvente, es débil? La autora es consciente de que la respuesta no es muy agradable para el mundo subdesarrollado; afirmando que este es un tema no menor en América Latina y en el subdesarrollo en general. Apoyándose en Rodrik, (2007. P. 101), nos dice que la siguiente apreciación calibra la gravedad del problema:

La innovación en los países periféricos se ve trabada no tanto por el lado de la oferta como por el lado de la demanda; las innovaciones requeridas para configurar una nueva estructura de las economías de bajos ingresos no se ven perjudicadas principalmente por la falta de cientíicos e ingenieros capacitados, la ausencia de laboratorios de I+D o la inadecuada protección de la propiedad intelectual sino por la falta de demanda proveniente de sus usuarios potenciales en la economía real, los empresarios. Y a su vez la demanda de innovaciones es baja porque los empresarios estiman que las nuevas actividades ofrecen baja rentabilidad. (Rodrik, 2007, p. $6)$.

Ahora bien, de lo argumentado se puede entender con claridad que la relevancia de la investigación académica, y muy en particular la de la investigación universitaria-que en América Latina da cuenta mayoritaria de lo descrito lineas arriba-, está ligada a una característica estructural del desarrollo productivo y podríamos también agregar social de la región. No es tan simple entonces agregar relevancia por cambios en la orientación de la investigación, como reiteradamente se reclama. El problema es sistémico, de modo que trabajar desde un solo lado no promete la obtención de mayores resultados (Sutz, 2014, p. 6).

\section{La relevancia de la investigación y el "para quién" y "con quién" hacer investigación.}

Al respecto, Sutz nos dice que la cuestión de para quién es relevante la investigación, desde hace algunas décadas está ya fuertemente presente en el debate académico, asociada al "con quién" se organiza el proceso de producción de conocimiento; de manera que el mismo hecho de plantear la pregunta confirma que la investigación de calidad siempre mostrará la pertinencia de sus aplicaciones satisfaciendo requerimientos de diferentes "para quién". Entre estos para quién, vamos a centramos en un argumento que consideramos relevante en el hilo de este apartado: 
La tercera misión de la universidad. Argumenta la autora que recientemente, en el marco de las discusiones en torno a la "tercera misión" de la universidad, se plantean nuevas orientaciones para la investigación universitaria intimamente vinculadas a la cuestión del "para quién".

Una de ellas, presente en América Latina y que reconoce inspiración en el movimiento de la Reforma de Córdoba, plantea que la investigación universitaria incorpore a su agenda problemas generales del desarrollo y, muy en particular, problemas que afectan a las partes más postergadas de la población (Herrera, 1979). Universidades con esa orientación pueden denominarse "universidades para el desarrollo" (Arocena et al, 2008; Goransson y Brundenius, 2011). Por otra parte, un enfoque muy influyente es el de la "universidad empresarial" (Etzkowitz et al, 2000), donde el "para quién" está conformado por el desarrollo económico y muy en particular sus agentes más directos, las empresas. Esa orientación tiene como consecuencia -y también como causa- la obtención del financiamiento necesario para trabajar, que crecientemente superaría las posibilidades del erario público, así como la obtención de una legitimidad derivada de la utilidad evidente, pues alguien paga por ellos, de los resultados de la investigación universitaria (Sutz, 2014, p. 8).

Esta problemática introduce un factor muy importante, que no es común que sea percibido con claridad en América Latina, ni muy tenido en cuenta en los sistemas universitarios. En estos, como dijimos en otro apartado, porque nuestras universidades ejercen principalmente la función académica más que la de investigación (pertinente/relevante y de calidad).

En este sentido, nuestra autora sostiene que la respuesta "crecimiento económico y directo interés empresarial" a la pregunta del para quién es relevante la investigación universitaria, lleva a ubicarnos en los modelos que existen en la transformación estructural en la universidad. Refiriendo que las instituciones donde

...por más estilizada y por tanto imprecisa que resulte la descripción, más bien se cultivaba el ethos mertoniano como ideal normativo, son bien diferentes de aquellas donde lo que predomina normativamente es otro ethos, el descrito por Ziman, donde quizá la mayor transformación sea el pasaje de la puesta en común del conocimiento generado a su apropiación privada con la consiguiente restricción de su uso libre (Merton, 1977; Ziman, 2002). Esta transformación, reconocible en su forma más acabada en el mundo anglosajón y especialmente en los Estados Unidos, ha sido denominada "capitalismo aca- 
démico" (Slaughter y Rhoades, 2004). Cabe notar que sólo puede haber capitalismo académico real -más allá de la opinión que sobre él se tenga- allí donde hay demanda empresarial por conocimiento; donde no la hay o es estructuralmente débil, la universidad que se pretende empresarial ni obtiene resultados utilizados por las empresas ni logra legitimidad/relevancia para su investigación (2014, p. 9).

Ahora seguiremos discurriendo sobre la pertinencia, tomando como referencia la opinión autorizada del profesor emérito de la Universidad de Chulalongkorn (Tailandia), Charas Suwanwela, quien en una conversación mantenida con el Secretariado de la GUNI (Global University Network for Innovation), habló acerca del rol que deben asumir las universidades de los países en vías de desarrollo en la investigación con pertinencia local y global para cubrir sus necesidades. Estas opiniones las hacemos extensivas a la universidad peruana en lo que sea propio, por ser foco de nuestro interés en el presente artículo y además por ser una universidad, también, en vías de desarrollo. Glosemos algunos aspectos de esta entrevista.

A la pregunta ¿Cuáles son los criterios de pertinencia que debería tener la investigación en los países en vías de desarrollo? El profesor Suwanwela sostiene que la pertinencia, aquí, está en que la investigación sea útil para el país; pues cada país, incluso los más pobres, debe llevar a cabo algún tipo de investigación para resolver sus propios problemas. Él hace notar que no solo hay que realizar investigaciones prístinas (que nunca las hay), sino también "cómo se puede transferir el conocimiento existente en el mundo para utilizarlo en el entorno local. Sabemos que en la actualidad existe una enorme base de conocimiento global a la que es posible acceder a través de Internet, pero en muchos países en vías de desarrollo no se utiliza en absoluto, ni para el beneficio de las personas ni en particular para la mayoría de ellas". Y esto se agrava cada vez más porque el los países no intercambian conocimiento.

Seguidamente, propone que la investigación en los países en vías de desarrollo debería realizarse, inicialmente, en el ámbito local para, después, aplicar la investigación disponible en el ámbito mundial, y no a la inversa. Transcribo el ejemplo usado por ser muy clarificador:

Por ejemplo, si queremos aplicar nuevos cultivos a la tierra, necesitaremos conocer las propiedades del suelo. Si lo pensamos detenidamente, este tipo de investigación no se puede publicar, o sólo se puede publicar en el ámbito local, porque sólo es relevante alli. Los resultados de este tipo de investigación no se podrian publicar a nivel internacional o en publicaciones que sirven para evaluar la calidad de la universidad a nivel mundial".

Es decir, nos recomienda que la universidad sea verdaderamente rele- 
vante para el país, en lugar de preocuparse en seguir la tendencia global y aplicar criterios que sirven para evaluar una universidad con criterios globales. Solamente así una universidad, como la peruana, hará el trabajo que corresponde a las necesidades de las personas de nuestro país, de manera que se sirva a la población de este país.

En relación a qué tipo de investigación debemos hacer, el ilustre profesor cree que la investigación básica es importante es del parecer que para resolver muchos de los problemas 1ocales se necesita mucha investigación básica, de modo que no sólo sería relevante la investigación aplicada. Precisamente aquí radica una de nuestros sesgos epistemológicos en la investigación nacional (y tercermundista), la desvalorización de la investigación básica ¿por ser no rentable?, afirmando que existe un tipo de investigación que es necesaria en el país y que las universidades no están llevando a cabo. Señala que "la pertinencia tiene un reverso también y, por tanto, las universidades deben considerarla desde todos los ángulos, los positivos y los negativos. Ése debería ser el modo de diseñar la política de investigación de la universidad".

Ante la pregunta de qué cambios habría que introducir para que las instituciones de educación superior produjeran conocimiento relevante, el Dr. Suwanwela opina que si priorizamos la investigación nacional “...es necesario aceptar los valores que identifican a esa nación y las necesidades que hay que resolver en el país". Es decir, la investigación en la universidad debe ser pertinente a las necesidades nacionales, tanto al nivel de necesidades sociales como de necesidades de conocimiento científico.

Además, Suwanwela enfáticamente nos recomienda que nosotros, como países en vías de desarrollo, debemos llevar a cabo investigación de primer nivel, porque, de lo contrario, los países desarrollados seguirán controlando el precio de los productos creados a partir de los resultados de la investigación que realizan. "De hecho, cuando un país en vías de desarrollo necesita conocimiento o tecnología para cubrir una necesidad, debe pagar un precio que, en ocasiones, no puede permitirse. Esto ocurre en sectores tan importantes como la medicina o la farmacia".

\section{PARTE II}

\section{La pertinencia de las tesis de inves- tigación universitarias}

La conciencia sobre la urgente necesidad de una educación superior con mayores niveles de pertinencia, es uno de los grandes aportes que hizo la Conferencia Mundial sobre Educación Superior desarrollada en París del 5 al 8 de octubre de 1998 (UNESCO).

Para Aguilera y Pino (2015), la pertinencia es un concepto relacional, que vincula o conecta la medida de la respuesta social a las demandas y necesidades de una sociedad, comunidad o institución. Ella se refleja en 
todas las actividades de lo humano, en todos sus planos: individual, social, profesional, y otros.

Sobre la Educación Superior, los autores consideran que es un bien público social, un derecho humano y universal y un deber del Estado. La universidad, por su propio cometido, prepara a los nuevos profesionales, incrementa el conocimiento y actúa de foco clarificador de futuras opciones axiológicas y socio-laborales, a la vez que es influida por las nuevas lineas e instrumentos tecnológicos que la sociedad del conocimiento ha desarrollado (p.133).

Parafraseando a Aguilera y Pino, en esta perspectiva surge la pregunta puntual acerca de en qué medida el objeto de estudio valorado por las tesis defendidas en la universidad responden a las necesidades de la sociedad, las instituciones y sectores de cada carrera profesional del país. De aquí se sigue que todas las tesis deberán concebirse incluyendo los sistemas de conocimientos más necesarios y apropiados al contexto, a la actualidad y a la necesidad profesional-social demandada en su área de conocimiento para el país (p. 143). En esta argumentación no está demás resaltar que la Universidad está investida de una función social que identifica con claridad sus destinatarios sociales: el Estado, los empresarios, la sociedad civil, tanto personas naturales como jurídicas, que necesiten de sus servicios para atender sus problemas y necesidades sociales. Es lo que se conoce como "Pertinencia de la Educación
Superior", la cual es la encargada de este cometido. Por estas razones, los autores comparten la tesis de que: "La pertinencia de la Educación Superior debe evaluarse en función de la adecuación entre lo que la sociedad espera de las instituciones y lo que ellas hacen" (p. 144).

Consecuentes con la aceptación de la intrínseca convivencia entre universidad-sociedad y los retos a los que en disimiles campos se enfrentan las Universidades y que pudiéramos identificar como de relación "oferta demanda” (tratando el vínculo UniversidadSociedad), son una consecuencia de la dinámica de desarrollo del conocimiento en la actualidad.

\section{La investigación en las tesis univer- sitarias: compromiso intelectual, social e histórico con el país}

Cuando decimos que una investigación (léase una tesis universitaria) debe ser pertinente -entre los variados usos y significados del término- es porque consideramos que es oportuna y conveniente. Es decir, se toma en consideración un tema-problema que realmente plantee objetivos con cuyo alcance se pueda aportar y argumentar el sentido que el investigador pretende alcanzar.

Si decimos que una investigación es relevante es porque está vinculada con un tema de importancia (relevancia), ya sea social, científico, político, empresarial, etc.; y además tiene características únicas que lo diferencian de otros temas de su misma clase. 
Tratándose de las tesis de grado o de posgrado, éstas deben procurar la imbricación de estos conceptos en la investigación, es decir, la investigación, además de ser oportuna y conveniente, debe estar relacionada con un temaproblema importante que aporte en sus resultados, nuevos conocimientos y posibles soluciones en un campo de interés. Esto, en la medida que la investigación ayuda a mejorar el estudio de temas concretos de la realidad y permite establecer contacto con la misma a fin de conocerla mejor; obteniendo con ella un estímulo para la actividad intelectual y creadora. Además, ayuda a establecer mecanismos de solución de problemas y, con ello, enfatiza el análisis crítico de diversos fenómenos en la realidad social y/o natural del país.

En resumidas cuentas, los tesistas deben ser conscientes de que en sus investigaciones deben abordar temas de contenidos relevantes y pertinentes, pues dichas investigaciones no son meros ejercicios retóricos y superfluos para pasar la prueba de "blanqueo" de requisitos metodológicos y administrativos. Ellos deben tener bien en claro que sus investigaciones son parte y producto de un entramado social e histórico con el que está comprometido, en la medida que el resultado de su investigación podrá contribuir directamente con la prosperidad del conocimiento individual y colectivo y no se está dando vueltas en círculos en temas que ya han sido analizados a profundidad. (Blog: dearmnemonic. blogspot.com/2011/05/la-pertinencia-y-la-relevancia-de-la.htm).

\section{Relevancia y validación de la inves- tigación de tesis}

La polisemia del término pertinencia/relevancia permite adecuar sus diversas acepciones a diversos miradas sobre el asunto; así por ejemplo, diremos que relevancia es la importancia de la investigación que se aporta para la obtención de nuevos conocimientos. Las preguntas que pueden hacerse para determinar la relevancia en el caso de tesis universitarias, son: ¿Para qué sirve? ¿A quiénes les servirán los nuevos conocimientos? ¿Cómo y a quiénes ayuda?

Es que la relevancia de la investigación señala relación con algo relativamente simple, que en la literatura de metodología de la investigación más estandarizada significa el justificar las razones que motivan la investigación; en términos más simples aún, debe explicar claramente por qué debe hacerse esta investigación, por qué es importante hacerla y qué se podría perder de no hacerla. La relevancia de la Investigación, entendida como las justificaciones de una tesis o investigación, es exponer las razones por las cuales se quiere y se debe realizar. Qué duda cabe que toda investigación debe realizarse con un propósito definido. Debe explicar por qué es conveniente la investigación y qué o cuáles son los beneficios que se esperan con el conocimiento obtenido. El investigador tiene que saber "vender la idea" de la investigación a realizar, por lo que deberá acentuar sus argumentos en los beneficios a obtener y en los usos 
sociales que se le dará al conocimiento. Aventuremos algunos criterios elementales para comprender la relevancia/pertinencia:

1. Conveniente. En cuanto al propósito académico o la utilidad social, el sentido de la urgencia. Para qué servirá y a quién le sirve.

2. Relevancia social: Trascendencia, utilidad y beneficios. ¿Cuál es su trascendencia para la sociedad?, ¿quiénes se beneficiarán con los resultados de la investigación?, ¿de qué modo? En resumen, ¿qué alcance social tiene?

3. Implicaciones prácticas: ¿Realmente tiene algún uso el producto de la investigación? ¿Ayudará a resolver algún problema práctico?, ¿tiene implicaciones trascendentales para una amplia gama de problemas prácticos?

4. Valor teórico: ¿Se va a cubrir algún vacío del conocimiento en el campo científico del tesista? Con la investigación ¿̇se podrán generalizar los resultados a principios más amplios?, ¿la información que se obtenga puede servir para comentar, desarrollar o apoyar una teoría?, ¿se podrá conocer en mayor medida el comportamiento de una o de diversas variables o la relación entre ellas?, ¿ofrece la posibilidad de una exploración fructífera de algún fenómeno?, ¿qué se espera saber con los resultados que no se conociera antes?, ¿puede sugerir ideas, recomendaciones o hipótesis a futuros estudios?

5. Utilidad metodológica: ¿Se va a crear y/o utilizar algún modelo nuevo y novedoso para el campo especiali- zado del tesista destinado a obtener y recolectar información? La investigación, ¿puede ayudar a crear un nuevo instrumento para recolectar o analizar datos?, ¿ayuda a la definición de un concepto, variable o relación entre variables?, ¿pueden lograrse con ella mejoras en la forma de experimentar con una o más variables?, ¿sugiere cómo estudiar más adecuadamente una población? (Glikowski, 2010).

¿Cuál es el estado de la investigación científica en la universidad peruana?

¿Los profesores de las universidades en nuestro medio realizan investigación científica acorde con lo que pudiéramos llamar relevante a nivel de universidad? La respuesta parece ser muy pesimista pues la mayoría de los docentes universitarios no investigan. De unos 56.626 docentes que trabajan en las universidades del país, públicas y privadas (SUNEDU, 2017), en el 2015, el total de personal dedicado a generar I+D fue de 5 mil 408 personas: 62.4 por ciento declaró tener la categoría de investigador (3,374 personas), 22.1 por ciento de técnicos y 15.5 por ciento restante tuvo la categoría de otro personal de apoyo. Esta realidad, llevada a nivel internacional, apunta a que el Perú se encuentra rezagado en número de investigadores. Se estimó que por cada mil integrantes de la PEA hay solo 0.2 investigadores, porcentaje que se ubica por debajo del promedio de América Latina y El Caribe: 1.3, y muy lejos del promedio de los países de la OCDE: 12.7. (CONCYTEC, 2016). 
En otra mirada del problema, en Latinoamérica, con pocas excepciones entre las que no nos encontramos, las universidades concentran su quehacer en la docencia. Si focalizamos nuestra realidad, constatamos que la creación excesiva de universidades privadas en los últimos tiempos ha incrementado preocupantemente la cantidad de "universidades de tiza y pizarra", universidades que no desarrollan investigación, que solo transmiten conocimientos. Como lo señalan Lemansson y Chiappe (1999) refiriéndose a Venezuela, consideración aplicable a nuestra realidad, "existe un número insuficiente de universidades en las cuales se practican actividades de investigación seria".

Parafraseando a Mayz y Pérez (1997), también debemos dejar esclarecido que esta situación lamentable encuentra sus causa en la precariedad de recursos que le asigna el Estado; pero aun así, con tan pobres recursos, las universidades públicas aportan un elevado porcentaje, más del $65 \%$ de los productos de investigación (especialmente publicaciones y algunas escasas patentes) en la producción nacional de ciencia y tecnología. El aporte de las universidades privadas y de las empresas es muy bajo y demuestra el poco interés por las actividades de investigación, en contraste con lo que sucede en los países capitalistas desarrollados, donde las empresas privadas realizan grandes inversiones traducidas en publicaciones y, especialmente, en patentes.

$\mathrm{Si}$ las cifras arriba anotadas no son optimistas, entonces es válida la pregunta ¿para qué hacer investigación científica en las universidades? Porque de esta manera podemos esperar que la universidad contribuya al desarrollo del país, con el compromiso de atender sus necesidades, problemas y carencias, a través de la formación de profesionales integros y debidamente calificados.

\section{La Universidad y la producción cientifica para la formación de recursos humanos}

Compartimos totalmente con los autores referidos que la necesidad de investigar en las universidades se justifica con la preparación de individuos con habilidades y conocimientos nuevos que tengan una formación académica adecuada en un mundo en acelerado desarrollo, es decir, al ritmo de los tiempos. No puede ser de otra manera, porque la sociedad que vivimos requiere capital humano para resolver sus problemas más inmediatos; y aquí radica la auténtica misión de la universidad: preparar y acrecentar ese capital. ¿Y cómo? Pues, fundamentalmente con la ciencia; por eso es necesario cultivarla y desarrollarla. Solo ella nos proporciona la materia prima más valiosa que hoy existe: el conocimiento; que además es el capital más seguro y rentable de la sociedad.

Sin embargo, respecto al tipo de conocimiento que debe producir la universidad y que tradicionalmente la identifica con la investigación básica, predominancia de muy largos años, comienza a ser cuestionada por los 
elevados costos y porque para muchos ésta no aporta resultados "útiles", "rentables", capaces de producir recursos para las universidades. Esta es una variable interviniente que ejerce fuerte presión sobre los investigadores para que realicen investigación aplicada, lo que ha influido para que muchos científicos de elevado nivel se dediquen a actividades que responden solo a la necesidad de una investigación rentable y a la producción de ingresos en trabajos rutinarios, la mayor parte de las veces relacionadas con la prestación de servicios (Mayz, p. 1997). De aquí se sigue que lo importante, entonces, no es distinguir entre conocimiento básico o aplicado, universal o local, útil o inútil, pertinente o impertinente, sino entre civilización y barbarie (Scharifker, 1998; Citado por Mayz y Pérez, 1997).

Retomando el hilo de nuestra argumentación, es importante recordar que solo mediante la investigación se podrá formar el recurso humano a nivel de especialistas con la alta calidad que el país requiere. Del mismo modo, se debe reconocer que la investigación es de importancia vital en los estudios de posgrado; no es posible tener egresados de alto nivel si no se investiga. Y esto es una verdadera problemática en la academia peruana, como veremos en el siguiente parágrafo.

\section{E1 posgrado en las universidades peruanas}

No es una novedad reconocer la persistencia en el reclamo que hace la sociedad a la universidad peruana pidiéndole una mayor relación con la realidad del país; reclamo, aparentemente sin mucha trascendencia en el trajín de la vida y vaivenes que capturan la preocupación de las gentes. Sin embargo, su atención no es sencilla, pues para mejorar esta relación resulta indispensable, primero, hacer un esfuerzo por integrar y vincular estrechamente la formación profesional, el posgrado y la investigación científica, tecnológica y humanística en las universidades.

En un contexto de prácticas cerradas es urgente dar mayor transparencia a las tareas académicas y científicas en las instituciones públicas, pues nada justifica que la universidad, sobre todo estatal, continúe su aislamiento, y la escasa producción científica adquiera un rumbo ajeno a la realidad regional. En la medida que las tareas universitarias no sean suficientemente visibles, la sociedad tendrá pleno derecho a cuestionar el quehacer institucional.

En el editorial del Boletín CSI- $\mathrm{N}^{\circ}$ 46, 2002 de la UNMSM, se afirma que en una concepción más amplia, el nivel de la formación profesional universitaria debería estar ligado a los niveles de investigación científica, tecnológica y humanística e, inclusive, a los niveles de posgrado. En este contexto, pone de relieve el rol del profesor universitario como el elemento básico en la formación profesional; entonces, éste en ningún caso puede estar exento del quehacer cotidiano orientado a la investigación básica, aplicada o de 
desarrollo experimental. De lo que se deduce que es necesario que el profesor universitario pueda pasar siempre renovadas experiencias de investigación a los estudiantes de pregrado (la investigación formativa) y posgrado (UNMSM, 2002).

Pero para crear un programa de posgrado, la investigación debe estar consolidada, ésta debe ser el eje del currículo, lo que permitiría enriquecer la discusión y la creación de conocimientos para generar un cambio de actitud en el posgraduando. En este punto, Mays y Pérez (1997), además, nos señalan que es conveniente tener muy en cuenta diferenciar dos etapas en el proceso de formación del estudiante. La primera, de ampliación de los conocimientos, mediante cursos. La segunda, de iniciación en la investigación científica, especialmente en el desarrollo de la tesis. Pero, como hemos insistido en los apartados anteriores, tesis con problemas pertinentes y relevantes para el país y el conocimiento científico.

Por otro lado, en la formación del posgrado es de resaltar el rol muy importante que presta la tutoria. Es decir, en lo referente a las investigaciones de tesis lo fundamental es el tema-problema, no cabe duda; a éste hay que sumarle la presencia de un buen tutor como algo esencial. Mayz y Pérez (citando a Di Prisco, 1991), insisten en que un buen tutor enseña más con su ejemplo que con clases o conferencias. Éste es uno de los aspectos de la actividad creativa en la ciencia que vale la pena tener siempre presente, ya que la investigación es un oficio que se apren- de al lado de un maestro. Es imposible convertirse en un investigador leyendo sobre cómo se hace la ciencia o leyendo libros de epistemología; por ello, la creencia en la existencia de un solo método científico es un mito creado por los que no hacen ciencia. En realidad, cada investigador tiene su "método", (Véase Feyerabend), que trasmite mediante la práctica a sus alumnos, quienes lo modifican, lo mejoran y lo actualizan.

En este marco cabe preguntarse ¿Cómo entender entonces que se permita la creación de posgrados en universidades sin trayectoria en la investigación?, lo cual nos lleva, otra vez, a una realidad muy preocupante en la universidad peruana, pues la calidad de los posgrados, con excepciones, es muy débil; precisamente, porque el ethos científico Mertoniano no es su esencia sino más bien el ethos mercantil o de mera meritocracia profesional, como lo confirma el Editorial del Boletín CSI- No 46: Según información de la Asamblea Nacional de Rectores (ANR), en el Perú, actualmente hay 350 programas de maestría y 66 programas doctorales, es decir, 416 programas de posgrado. Por otra parte, nuestra producción científica en la forma de artículos publicados en revistas de calidad e impacto en el mundo científico escasamente se aproxima a los 225 artículos por año durante los 4 últimos años. No obstante, la conexión de la investigación con los numerosos programas es mínima. Esta proliferación de maestrías y docto- 
rados en nuestro país debe más a la exigencia de los centros de trabajo, a las necesidades personales de los candidatos, que a un interés académico y del país por el contrario con cuadros altamente calificados. También, hay que ser objetivos en reconocer, que los posgrados se han convertido en una fuente de generar recursos propios para no pocas universidades. La oferta y demanda ha generado un mercado muy activo, tenemos nombres de maestrías y doctorados que, en muchos casos, no corresponden a las nomenclaturas y denominaciones internacionales. (UNMSM, 2002).

Como el lector notará, la información proporcionada corresponde a varios años atrás, pues no hay información actualizada sino hasta el año 2010, que es nuestra referencia:

\section{Programas de posgrado según tipo de universidad}

De las 90 universidades con carreras de CINTEC, 44 cuentan con programas de maestrias en este ámbito y 21 con programas doctorales. Asimismo, se observa que el $74,3 \%$ de las universidades públicas cuentan con programas de maestría en CINTEC mientras que solo el $29,5 \%$ de universidades privadas ofrece estos programas. Las diferencias son más evidentes a nivel de programas de doctorado, dado que solo el $11 \%$ de universidades privadas ofrecen estos programas en comparación con el $40 \%$ de universidades públicas. (CONCYTEC, 2014).

Se pude advertir que esta información, a diferencia de la proporcionada por la ANR, solamente ha tenido en cuenta las carreras de CINTEC. En este caso, se consideran como carreras de Ciencias, Ingeniería y Tecnología (CINTEC) a aquellas relacionadas a ingeniería y tecnología (con excepción de ingeniería civil e industrial), ciencias de la salud (con excepción de medicina, enfermería y obstetricia), ciencias biológicas y ambientales, ciencias agrícolas y ciencias básicas (CONCYTEC, 2014). 


\section{REFERENCIAS BIBLIOGRÁFICAS}

Acuña, C., Valenzuela, P. La pertinencia en la investigación. Disponible en: http://148.231.9.38/JG/foro/pdf/25.pdf. Consultado: 15-7-2016

Aguilera, L., Pino, Y. (2015). Metodología para la evaluación de la pertinencia e impacto de las tesis defendidas en la facultad de derecho de la universidad de Holguin. Revista de Ciencias Sociales - No 66 (2015) - Universidad de Valparaíso - ISSN 0716-7725 - Valparaíso, Chile - revistas.uv.cl/index.php/ rcs/article/view/210. Disponible en: dearmnemonic.blogspot.com/2011/05/ la-pertinencia-y-la-relevancia-de-la.htm.

CONCYTEC - Consejo Nacional de Ciencia, Tecnología e Innovación Tecnológica (2014). Situación de la formación de capital humano e investigación en las universidades peruanas. II Censo Nacional Universitario 2010. Edición mayo 2014.

CONCYTEC - Consejo Nacional de Ciencia, Tecnología e Innovación Tecnológica (2016). I Censo Nacional de Investigación y Desarrollo a Centros de Investigación.

Derrida, J. (2002). Universidad sin condición. Minima Trotta, España

Garrocho, C. y Segura L. La pertinencia social y la investigación científica en la universidad pública mexicana. CIENCIA ergo sum, Vol. 19-1, marzo-junio 2012. Universidad Autónoma del Estado de México, Toluca, México. Pp. 24-34. Disponible en: http:/ /www.redalyc.org/pdf/104/10422917003.pdf

González, F. Algunas ideas para clarificar el significado de la pertinencia de la investigación. Disponible en: https://www.academia.edu/12178185/ALGUNAS_ IDEAS_PARA_CLARIFICAR_EL_SIGNIFICADO_DE_LA_PERTINENCIA_DE_LA_ INVESTIGACI\%C3\%93N

González, E. La investigación universitaria y su transformación epistemológica en el contexto latinoamericano. Disponible en: www.umariana.edu.co/ojseditorial/index.php/unimar/article/view/172 Consultado: 13-4-2017.

Sutz, J. Calidad y relevancia en la investigación universitaria: apuntes para avanzar hacia su convergencia. Revista CTS, $\mathrm{n}^{\circ} 27$, vol. 9, Septiembre de 2014. Disponible en: http://www.revistacts.net/volumen-9-numero-27/286dossier / 620-calidad-y-relevancia-en-la-investigacion-universitaria-apuntespara-avanzar-hacia-su-convergencia. Consultado, 29-5-2017.

Suwanwela, Charas (2008). Investigación con pertinencia local y global. Global University Network for Innovation. Boletín electrónico de la GUNI. 22 de diciembre de 2008. Disponible en: http://noticias.universia.net.co/vidauniversitaria/noticia/2008/12/22/239418/investigacion-pertinencia-localglobal.html. Consultado: 9-6-2017.

UNMSM. Consejo Superior de Investigaciones (2002). "La universidad y la producción científica”. Boletín CSI-N46, 2002. Editorial. Disponible en: http:// sisbib.unmsm.edu.pe/BibVirtual/Publicaciones/consejo/2002_n46/indice. htm Consultado: 12-8-2017 